\title{
ANALYTIC PERTURBATION PRESERVES DETERMINACY OF INFINITE INDEX
}

\author{
PETER YUDITSKII
}

In this note we give an answer to a question of Christian Berg. To formulate the question and our result we need some definitions and notation.

Let $\mu$ be a positive measure on the real axis having moments of every order. A measure $\mu$ is determinate if no other measure has the same moments as those of $\mu$, otherwise $\mu$ is indeterminate. By $\mathscr{M}_{0}$ we denote the set of measures having a finite number of real points as support. Following [2-5] (see also [8]), we say that a measure $\mu$ has an infinite index of determinacy if, for any measure $\mu_{0} \in \mathscr{M}_{0}$, the measure $\mu+\mu_{0}$ is determinate. The question posed by Berg is the following. Suppose that the measure $\mu$ has infinite index of determinacy and the measure $\nu$ has a compact support. Is it true that the measure $\mu+\nu$ is determinate?

Here, we will give a positive answer to this question. Moreover, we will prove a stronger result.

One of the most known sufficient determinacy condition on a measure $\nu$ is the following [6, Theorem 5.2]

$$
\int_{-\infty}^{\infty} e^{\epsilon|t|} d \nu<\infty, \quad \text { for some } \epsilon>0
$$

In this case, the Fourier transform of the measure $\nu$

$$
\phi(z)=\int_{-\infty}^{\infty} e^{i t z} d \nu,
$$

is analytic in the strip $|\operatorname{Im} z|<\epsilon$. The main result of this note is the following:

THEOREM. Suppose that $\mu$ has infinite index of determinacy and $\nu$ satisfies condition (1). Then the measure $\mu+\nu$ is determinate.

A counterpart of this theorem in the setting of the Bernstein approximation problem was recently proved by Sodin [8]. However, his proof is based

Received October 27, 1997. 
on the de Branges criterion of density of polynomials in weighted spaces with uniform norm which apparently does not work in the case of $L^{2}$-norm. As usual, the question in $L^{2}$-norm can be treated by relatively simpler tools. Our proof is based on the following lemma.

Lemma. Let $\tilde{\mu}$ be an indeterminate measure and let $\nu$ satisfy (1). Let $H=\operatorname{clos}_{L_{d \tilde{O}}^{2}} \mathscr{P}$ be the closure of the polynomials in the space $L_{d \tilde{\mu}}^{2}$. Then the quadratic form $\int_{-\infty}^{\infty} \bar{Q} P d \nu$ defines a (bounded positive) operator $A$ in $H$

$$
\int_{-\infty}^{\infty} \bar{Q} P d \nu=\langle A P, Q\rangle
$$

of the trace class: i.e. $\operatorname{tr} A<\infty$.

Proof. Let $\left\{P_{n}\right\}$ be the system of orthonormal polynomials with respect to the measure $\tilde{\mu}$. They form an orthonormal basis of the space $H$. The matrix elements of the operator $A$ with respect to this basis are of the form

$$
a_{m, n}=\left\langle A P_{n}, P_{m}\right\rangle=\int_{-\infty}^{\infty} \overline{P_{m}} P_{n} d \nu .
$$

In particular,

$$
a_{n, n}=\int_{-\infty}^{\infty} P_{n}^{2} d \nu
$$

and we are going to prove that

$$
\sum_{n=0}^{\infty} a_{n, n}=\sum_{n=0}^{\infty} \int_{-\infty}^{\infty} P_{n}^{2} d \nu<\infty
$$

It follows that the matrix $\left(a_{m, n}\right)$ determines a positive bounded operator $A$ of trace class in $H$.

A well known theorem of M. Riesz (see, for example, [1, Ch. II, §4]) says that in the case of indeterminacy the series $\sum_{n=0}^{\infty} P_{n}^{2}$ converges and satisfies the following estimate

$$
\sum_{n=0}^{\infty} P_{n}^{2}(t) \leq C(\delta) e^{\delta|t|}, \quad \text { for any } \delta>0 .
$$

Making use of (1), we obtain

$$
\sum_{n=0}^{\infty} a_{n, n}=\sum_{n=0}^{\infty} \int_{-\infty}^{\infty} P_{n}^{2} d \nu=\int_{-\infty}^{\infty} \sum_{n=0}^{\infty} P_{n}^{2} d \nu \leq C(\delta) \int_{-\infty}^{\infty} e^{\delta|t|} d \nu<\infty,
$$

completing the proof.

The following proposition yields the Theorem. 
Proposition. Let $\mu$ be a positive measure, let $\nu$ satisfy condition (1) and assume that $\tilde{\mu}=\mu+\nu$ is an indeterminate measure. Then there exists a measure $\mu_{0} \in \mathscr{M}_{0}$ such that $\mu+\mu_{0}$ is indeterminate.

Proof. Let $A$ be the operator in the space $H=\operatorname{clos}_{L_{d \tilde{\mu}}^{2}} \mathscr{P}$ associated to the measure $\nu$ as in the previous lemma. Since

$$
\langle(I-A) P, P\rangle=\int_{-\infty}^{\infty}|P|^{2} d(\tilde{\mu}-\nu)=\int_{-\infty}^{\infty}|P|^{2} d \mu,
$$

we have $(0 \leq) A \leq I$. Suppose that $\|A\|<1$. In this case the operator $I-A$ is invertible and we have

$$
\begin{aligned}
\int_{-\infty}^{\infty}|P|^{2} d \mu & =\langle(I-A) P, P\rangle \\
& \geq\left\|(I-A)^{-1}\right\|^{-1}\langle P, P\rangle=\left\|(I-A)^{-1}\right\|^{-1} \int_{-\infty}^{\infty}|P|^{2} d \tilde{\mu} .
\end{aligned}
$$

Let us use the criterion which follows from [1, Ch. II]: the measure $\tilde{\mu}$ is indeterminate if and only if the point-evaluation functional $P \mapsto P(z)$ is bounded in $L_{d \tilde{\mu}}^{2}$ : i.e.

$$
|P(z)|^{2} \leq C \int_{-\infty}^{\infty}|P|^{2} d \tilde{\mu} .
$$

As it follows from (2) and (3),

$$
|P(z)|^{2} \leq C \int_{-\infty}^{\infty}|P|^{2} d \tilde{\mu} \leq C||(I-A)^{-1} \| \int_{-\infty}^{\infty}|P|^{2} d \mu .
$$

And hence, due to this criterion, the measure $\mu$ is itself indeterminate.

Let now $\|A\|=1$. Since $\operatorname{tr} A<\infty$, the value $\lambda=1$ is just an isolated eigenvalue of finite multiplicity. Let us split the space $H$ into the orthogonal sum

$$
H=H_{0} \oplus H_{1},
$$

where $H_{0}=\operatorname{ker}(I-A)$. The operator $A$ has the following decomposition

$$
A=\left[\begin{array}{cc}
I_{H_{0}} & 0 \\
0 & A_{1}
\end{array}\right]: H_{0} \oplus H_{1} \rightarrow H_{0} \oplus H_{1},
$$

and, what is essential for us, $\operatorname{dim} H_{0}=n<\infty$ and

$$
|| A_{1} \|<1 .
$$

Let $\left\{f_{1}, \ldots, f_{n}\right\}$ be a system of entire functions which forms an orthonormal basis in $H_{0} \subset H$ (condition (3) readily yields that the elements of $H$ are entire functions). We state that the measure we need is any measure $\mu_{0} \in \mathscr{M}_{0}$ with support $\operatorname{supp}\left(\mu_{0}\right)=\left\{t_{1}, \ldots, t_{n}\right\}$ possessing the property 
ANALYTIC PERTURBATION PRESERVES DETERMINACY OF INFINITE INDEX 291

$$
\operatorname{det}\left|\begin{array}{ccc}
f_{1}\left(t_{1}\right) & \ldots & f_{n}\left(t_{1}\right) \\
\vdots & & \vdots \\
f_{1}\left(t_{n}\right) & \ldots & f_{n}\left(t_{n}\right)
\end{array}\right| \neq 0
$$

Linear independence of $f_{1}, \ldots, f_{n}$ guaranties that such a choice is always possible.

Let $\mu_{0}$ be a measure of this type. Introduce an operator $B$ from $H$ to the $n$ dimensional space $\mathrm{C}^{n}$ of the form

$$
B f=\left[\begin{array}{c}
\sqrt{\mu_{0}\left(t_{1}\right)} f\left(t_{1}\right) \\
\vdots \\
\sqrt{\mu_{0}\left(t_{n}\right)} f\left(t_{n}\right)
\end{array}\right], \quad f \in H .
$$

In this case,

$$
\begin{aligned}
\int_{-\infty}^{\infty}|P|^{2} d\left(\mu+\mu_{0}\right) & =\int_{-\infty}^{\infty}|P|^{2} d \tilde{\mu}-\int_{-\infty}^{\infty}|P|^{2} d \nu+\int_{-\infty}^{\infty}|P|^{2} d \mu_{0} \\
& =\left\langle\left(I-A+B^{*} B\right) P, P\right\rangle .
\end{aligned}
$$

As before, we just need to prove that the operator $I-A+B^{*} B$ is invertible.

Let us consider the block-decomposition of this operator:

$$
\begin{aligned}
I-A+B^{*} B & =\left[\begin{array}{cc}
I_{H_{0}} & 0 \\
0 & I_{H_{1}}
\end{array}\right]-\left[\begin{array}{cc}
I_{H_{0}} & 0 \\
0 & A_{1}
\end{array}\right]+\left[\begin{array}{l}
B_{0}^{*} \\
B_{1}^{*}
\end{array}\right]\left[\begin{array}{ll}
B_{0} & B_{1}
\end{array}\right] \\
& =\left[\begin{array}{cc}
B_{0}^{*} B_{0} & B_{0}^{*} B_{1} \\
B_{1}^{*} B_{0} & I_{H_{1}}-A_{1}+B_{1}^{*} B_{1}
\end{array}\right],
\end{aligned}
$$

where $\left[\begin{array}{ll}B_{0} & B_{1}\end{array}\right]$ is the block decomposition of the operator $B$.

A positive block-operator

$$
C=\left[\begin{array}{ll}
C_{11} & C_{12} \\
C_{21} & C_{22}
\end{array}\right]
$$

is invertible if and only if the operators $C_{11}$ and $C_{22}-C_{21} C_{11}^{-1} C_{12}$ are invertible (see e.g. [7]):

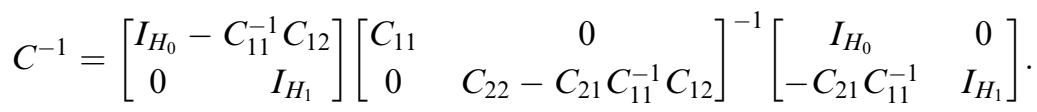

In our case, we need invertibility of the operators $B_{0}^{*} B_{0}$ and

$$
I_{H_{1}}-A_{1}+B_{1}^{*} B_{1}-B_{1}^{*} B_{0}\left(B_{0}^{*} B_{0}\right)^{-1} B_{0}^{*} B_{1}=I_{H_{1}}-A_{1} .
$$

In the basis $f_{1}, \ldots, f_{n}$, the matrix of the operator $B_{0}$ has the form 


$$
\left[\begin{array}{ccc}
\sqrt{\mu_{0}\left(t_{1}\right)} & & \\
& \ddots & \\
& & \sqrt{\mu_{0}\left(t_{n}\right)}
\end{array}\right]\left[\begin{array}{ccc}
f_{1}\left(t_{1}\right) & \ldots & f_{n}\left(t_{1}\right) \\
\vdots & & \vdots \\
f_{1}\left(t_{n}\right) & \ldots & f_{n}\left(t_{n}\right)
\end{array}\right],
$$

hence invertibility of $B_{0}$ is equivalent to (6). Invertibility of $I_{H_{1}}-A_{1}$ follows from (5). So, exactly as in (4), we have

$$
|P(z)|^{2} \leq C||\left(I-A+B^{*} B\right)^{-1} \| \int_{-\infty}^{\infty}|P|^{2} d\left(\mu+\mu_{0}\right)
$$

and this completes the proof.

ACKNOWLEDGEMENT. This work was done during author's short visit to Israel in Summer 1997 for participation in the conference dedicated to the Moshe Livŝic 80-th birthday. The author thanks Daniel Alpay, Victor Katsnelson and Victor Vinnikov who made this trip possible. The author thanks Mikhail Sodin for useful discussion and, in particular, for formulation, as a conjecture, the main result of this work.

\section{REFERENCES}

1. N. I. Akhiezer, The classical moment problem, Oliver and Boyd, Edinburgh 1965.

2. C. Berg and A. Duran, The index of determinacy for measures and $l^{2}$-norm of orthonormal polynomials, Trans. Amer. Math. Soc. 347 (1995), 2795-2811.

3. C. Berg, Indeterminate moment problems and the theory of entire functions, J. Comput. Appl. Math. (1995), 27-55.

4. C. Berg, Moment problems and polynomial approximation, Ann. Fac. Sci. Toulouse (1996), 9-32.

5. C. Berg and A. Duran, Measures with finite index of determinacy or a mathematical model for Dr. Jekyll and Mr. Hyde, Proc. Amer. Math. Soc. 125 (1997), 523-530.

6. G. Freud, Orthogonale polynome, Birkhäuser 1969.

7. M. G. Krein, The theory of self-adjoint extensions of semi-bounded Hermitian operators and applications, I, Mat. Sb. 20 (1947), 431-495. Russian.

8. M. Sodin, Which perturbations of quasianalytic weights preserve quasianalyticity? How to use de Branges' theorem, J. Analyse Math. 69 (1996), 293-309.

MATHEMATICAL DIVISION

INSTITUTE FOR LOW TEMPERATURE PHYSICS

KHARKOV

LENIN'S PR. 47, 310164

UKRAINE

E-mail: yuditskii@ilt.kharkov.ua 